\title{
A lower bound on opaque sets ${ }^{\star}$
}

\author{
Akitoshi Kawamura ${ }^{1}$, Sonoko Moriyama ${ }^{2}$, Yota Otachi ${ }^{3}$, and János $\mathrm{Pach}^{4}$ \\ 1 University of Tokyo, kawamura@is.s.u-tokyo.ac.jp \\ 2 Nihon University, moriso@chs.nihon-u.ac.jp \\ 3 Japan Advanced Institute of Science and Technology, otachi@jaist.ac.jp \\ 4 EPFL, Lausanne and Rényi Institute, Budapest, pach@cims.nyu.edu
}

\begin{abstract}
It is proved that the total length of any set of countably many rectifiable curves, whose union meets all straight lines that intersect the unit square $U$, is at least 2.00002. This is the first improvement on the lower bound of 2 established by Jones in 1964. A similar bound is proved for all convex sets $U$ other than a triangle.
\end{abstract}

\section{Introduction}

A barrier or an opaque set for $U \subseteq \mathbb{R}^{2}$ is a set $B \subseteq \mathbb{R}^{2}$ that intersects every line that intersects $U$. For example, when $U$ is a square, any of the four sets depicted in Figure 1 is a barrier. Note that some part of the barrier may lie outside $U$ (Figure 2), and the barrier need not be connected. This notion dates back at least to Mazurkiewicz's work in 1916 [11].

We are interested in "short" barriers $B$ for a given object $U$, and hence we restrict attention to rectifiable barriers $B$. By this we mean that $B$ is a union of countably many curves $\beta$, pairwise disjoint except at the endpoints, that each have finite length $|\beta|$, and the sum of these lengths converges. We call this sum the length of $B$ and denote it by $|B|$.

Finding the shortest barrier is hard, even for simple shapes $U$, such as the square, the equilateral triangle, and the disk [6, 9]. The shortest known barrier for the unit square is the rightmost one in Figure 1 w with length $2.638 \ldots$. This problem and its relatives have an extensive literature. See [6, 10] and the introduction of [5] for more history, background, and related problems.

The best known lower bound for the unit square has been 2, established by Jones in 1964 [8]. In general, for convex $U$, a barrier needs to have length at least half the perimeter of $U$ (we review a proof in Section 2):

Lemma 1. $|B| \geq p$ for any rectifiable barrier $B$ of a convex set $U \subseteq \mathbb{R}^{2}$ with perimeter $2 p$.

\footnotetext{
* The work presented here was supported in part by JSPS KAKENHI, by the ELC project (Grant-in-Aid for Scientific Research on Innovative Areas, MEXT, Japan), by OTKA under EUROGIGA projects GraDR and ComPoSe 10-EuroGIGA-OP-003, and by Swiss National Science Foundation Grants 200020-144531 and 200021-137574.
} 

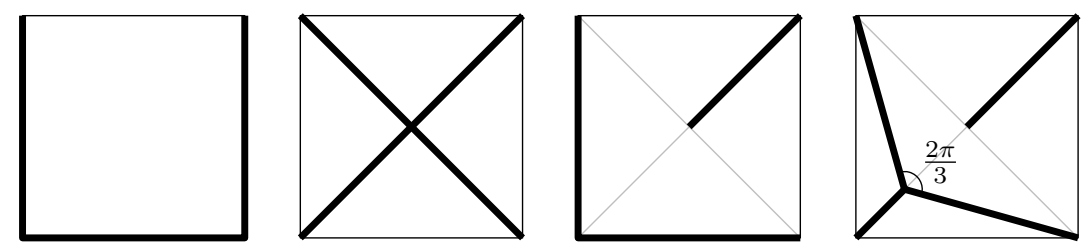

Fig. 1. Barriers (in thick lines) for the unit square. The first one (three sides) and the second one (diagonals) have lengths 3 and $2 \sqrt{2}=2.828 \ldots$, respectively. The third barrier consists of two sides and half of a diagonal, and has length $2+1 / \sqrt{2}=2.707 \ldots$ The last one is the shortest known barrier for the unit square, with length $\sqrt{2}+\sqrt{6} / 2=$ $2.638 \ldots$, consisting of half a diagonal and the Steiner tree of the lower left triangle.

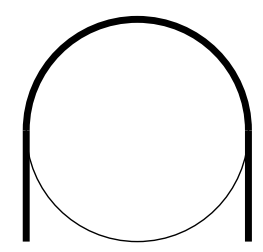

Fig. 2. A barrier (in thick lines) for a disk that is shorter than the perimeter. This is not the shortest one; see [6].

Thus, from the point of view of finding short barriers, the trivial strategy of enclosing the entire perimeter (or the perimeter of the convex hull if $U$ is a nonconvex connected set) gives a 2-approximation. See [4] and references therein for algorithms that find shorter barriers. The current best approximation ratio is $1.58 \ldots[5]$.

Proving a better lower bound has been elusive (again, even for specific shapes $U)$. There has been some partial progress under additional assumptions about the shape (single arc, connected, etc.) and location (inside $U$, near $U$, etc.) of the barrier [1, 3, 7, 10, 12, but establishing an unconditional lower bound strictly greater than 2 for the unit square has been open (see [4, Open Problem 5] or [3, Footnote 1]). We prove such a lower bound in Section 4

Theorem 2. $|B| \geq 2.00002$ for any rectifiable barrier $B$ of the unit square $\square$.

Dumitrescu and Jiang $\left[3\right.$ recently obtained a lower bound of $2+10^{-12}$ under the assumption that the barrier lies in the square obtained by magnifying $\square$ by 2 about its centre. Their proof, conceived independently of ours and at about the same time, is based on quite different ideas, most notably the line-sweeping technique. It will be worth exploring whether their techniques can be combined with ours.

Our proof can be generalized (Section 5): 


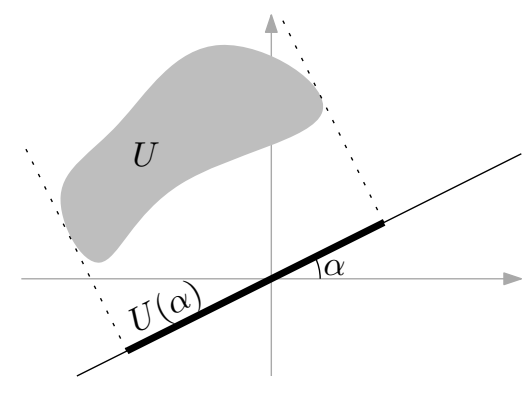

Fig. 3. The image $U(\alpha) \subseteq \mathbb{R}$ of $U$.

Theorem 3. For any closed convex set $U$ with perimeter $2 p$ that is not a triangle, there is $\varepsilon>0$ such that any barrier $B$ for $U$ has length at least $p+\varepsilon$.

Thus, the only convex objects for which we fail to establish a lower bound better than Lemma 1 are triangles.

The rest of this paper is structured as follows. In Section 2 , we present Jones' proof for Lemma 1. We also prove that instead of rectifiable barriers, it is sufficient to restrict our attention to barriers comprised of line segments. In Section 3 , we present three preliminary lemmas, analyzing some important special cases in which we can expect to improve on Jones' bound. The proof of one of these lemmas is postponed to Section 6. The three preliminary lemmas are combined in Section 4 to obtain our lower bound for the length of a barrier for the square (Theorem 2). In Section 5, we show how to generalize these arguments to other convex sets (Theorem 3). In the last section, we discuss a closely related question.

\section{Preliminaries: A general lower bound}

For a set $U$ and an angle $\alpha \in[0,2 \pi$ ) (all angle calculation will be performed modulo $2 \pi$ ), we write $U(\alpha) \subseteq \mathbb{R}$ for the image of $U$ projected onto the line passing through the origin and enclosing angle $+\alpha$ with the positive $\mathrm{x}$-axis, i.e.,

$$
U(\alpha)=\{x \cos \alpha+y \sin \alpha:(x, y) \in U\}
$$

(Figure 3). To say that $B$ is a barrier of $U$ means that $B(\alpha) \supseteq U(\alpha)$ for all $\alpha$.

For the discussion of upper and lower bounds on the length of a barrier, the following lemma says that it suffices to consider barriers that are a countable union of line segments. We call such a barrier straight.

Lemma 4 ([5, Lemma 1]). Let $B$ be a rectifiable barrier for $U \subseteq \mathbb{R}^{2}$. Then, for any $\varepsilon>0$, there exists a straight barrier $B_{\varepsilon}$ for $U$ such that $\left|B_{\varepsilon}\right| \leq(1+\varepsilon)|B|$.

Proof. Since the proof in [5] has a gap, we provide another proof. We will show that for any $\varepsilon>0$ and any curve $\beta$, there is a straight barrier $\beta^{\prime \prime}$ of $\beta$ of length 
$\leq(1+\varepsilon)|\beta|$. We can then apply this construction to each curve comprising $B$ to obtain the claimed straight barrier $B_{\varepsilon}$ of $U$.

If $\beta$ is already a line segment, we are done. Otherwise, the convex hull $H$ of $\beta$ has an interior point. Let $\beta^{\prime}$ be the curve obtained by magnifying $\beta$ by $1+\varepsilon$ about this point. Since the convex hull of $\beta^{\prime}$ contains the compact set $H$ in its interior, so does the convex hull of a sufficiently fine polygonal approximation $\beta^{\prime \prime}$ of $\beta^{\prime}$. This implies that $\beta^{\prime \prime}$ is a barrier of $\beta$.

By Lemma 4, we may focus attention on straight barriers: $U$ has a rectifiable barrier of length $<l$ if and only if it has a straight barrier of length $<l$.

As mentioned in the introduction (Lemma 1), it has been known that any barrier of a convex set must be at least half the perimeter. We include a short proof of this bound here, for completeness and further reference. See [2] for another elegant proof.

Proof of Lemma 1. By Lemma 4, we may assume that $B$ consists of line segments. We have

$$
|U(\alpha)| \leq|B(\alpha)| \leq \sum_{b}|b(\alpha)|=\sum_{b}|b| \cdot\left|\cos \left(\alpha-\theta_{b}\right)\right|
$$

for each $\alpha \in[0,2 \pi)$, where the sum is taken over all line segments $b$ that comprise $B$ without overlaps, and $\theta_{b}$ is the angle of $b$. Integrating over $[0,2 \pi)$, we obtain

$$
\int_{\alpha=0}^{2 \pi}|U(\alpha)| \mathrm{d} \alpha \leq \sum_{b}\left(|b| \cdot \int_{\alpha=0}^{2 \pi}\left|\cos \left(\alpha-\theta_{b}\right)\right| \mathrm{d} \alpha\right)=4 \sum_{b}|b|=4|B| .
$$

When $U$ is a convex set, the left-hand side equals twice the perimeter.

\section{Preliminary lemmas}

Note that Theorems 2 and 3 do not merely state the non-existence of a straight barrier $B$ of length exactly half the perimeter of $U$. Such a claim can be proved easily as follows: If $B$ is such a barrier, the inequality (3) must hold with equality, and so must (2) for each $\alpha$. Thus, the second inequality in 2 must hold with equality, which means that $B$ never overlaps with itself when projected onto the line with angle $\alpha$. Since this must be the case for all $\alpha$, the entire $B$ must lie on a line, which is clearly impossible.

The theorems claim more strongly that a barrier must be longer by an absolute constant. The following lemma says that in order to obtain such a bound, we should find a part $B^{\prime} \subseteq B$ of the barrier whose contribution to covering $U$ is less than the optimal by at least a fixed positive constant.

Lemma 5. Let $B$ be a barrier of a convex polygon $U$ of perimeter $2 p$. Then $|B| \geq p+\delta$ if there is a subset $B^{\prime} \subseteq B$ with

$$
\int_{\alpha=0}^{2 \pi}\left|B^{\prime}(\alpha) \cap U(\alpha)\right| \mathrm{d} \alpha \leq 4\left|B^{\prime}\right|-4 \delta .
$$



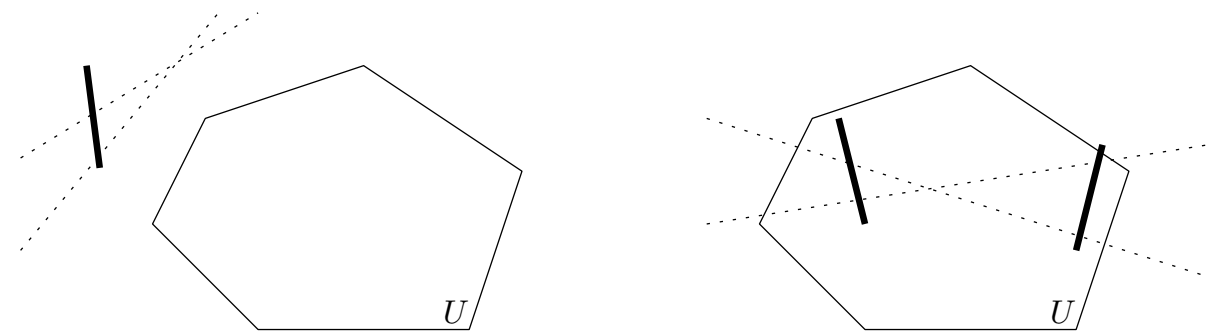

Fig. 4. Two wasteful situations. In the left figure, a barrier segment (thick) lies far outside the object $U$, which leads to significant waste because this segment covers in vain some lines (dotted) that do not pass through $U$; this is discussed in Lemma 6. In the right figure, there are two parts of the barrier (thick) that face each other, which also results in significant waste because they cover some lines (dotted) doubly; this is roughly the situation discussed in Lemma 7

Proof. For each $\alpha \in[0,2 \pi)$, we have $U(\alpha) \subseteq B(\alpha)$, and thus

$$
\begin{aligned}
|U(\alpha)| & =|B(\alpha) \cap U(\alpha)| \leq\left|\left(B \backslash B^{\prime}\right)(\alpha) \cap U(\alpha)\right|+\left|B^{\prime}(\alpha) \cap U(\alpha)\right| \\
& \leq\left|\left(B \backslash B^{\prime}\right)(\alpha)\right|+\left|B^{\prime}(\alpha) \cap U(\alpha)\right| .
\end{aligned}
$$

Integrating over $\alpha \in[0,2 \pi)$ and using the assumption (4), we get $4 p \leq 4 \mid B \backslash$ $B^{\prime}\left|+\left(4\left|B^{\prime}\right|-4 \delta\right)=4\right| B \mid-4 \delta$.

There are several ways in which such a "waste" can occur, and we make use of two of them (Figure 4). The first one is when there is a significant part of the barrier that lies far outside $U$, as described in the following lemma:

Lemma 6. Let $b$ be a line segment that lies outside a convex region $U$. Suppose that the set $A:=\{\alpha \in[0,2 \pi): U(\alpha) \cap b(\alpha) \neq \emptyset\}$ (of angles of all lines through $U$ and $b)$ has measure $\leq 2 \pi-4 \varepsilon$. Then

$$
\int_{\alpha=0}^{2 \pi}|b(\alpha) \cap U(\alpha)| \mathrm{d} \alpha \leq 4|b| \cos \varepsilon .
$$

Proof. We have

$$
\int_{\alpha=0}^{2 \pi}|b(\alpha) \cap U(\alpha)| \mathrm{d} \alpha \leq \int_{\alpha \in A}|b(\alpha)| \mathrm{d} \alpha=|b| \cdot \int_{\alpha \in A}\left|\cos \left(\alpha-\theta_{b}\right)\right| \mathrm{d} \alpha \leq 4|b| \cos \varepsilon,
$$

where the equality in the last inequality is attained when $A=\left[\varepsilon+\theta_{b}, \pi-\varepsilon+\right.$ $\left.\theta_{b}\right] \cup\left[\pi+\varepsilon+\theta_{b}, 2 \pi-\varepsilon+\theta_{b}\right]$.

The second situation where we have a significant waste required in Lemma 5 is when there are two sets of barrier segments that roughly face each other:

Lemma 7. Let $\lambda \in\left(0, \frac{\pi}{2}\right), \kappa \in(0, \lambda)$ and $l, D>0$. Let $B^{-}$and $B^{+}$be unions of $n$ line segments of length $l$ (Figure 5) such that 


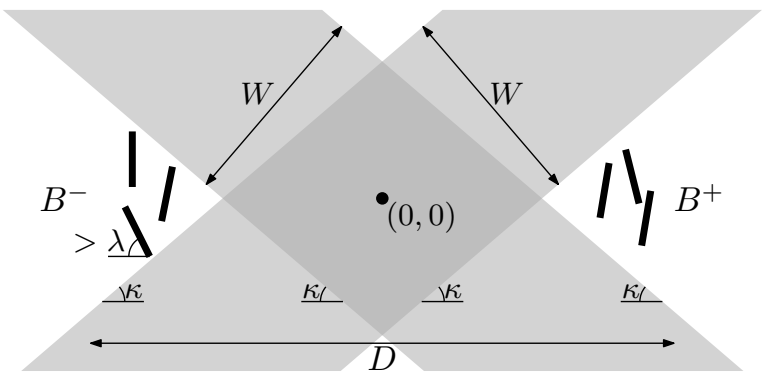

Fig. 5. Sets $B^{-}$and $B^{+}$(Lemma 7).

1. every segment of $B^{-} \cup B^{+}$makes angle $>\lambda$ with the horizontal axis;

2. $B^{-} \cup B^{+}$lies entirely in the disk of diameter $D$ centred at the origin;

3. $B^{-}$and $B^{+}$are separated by bands of angle $\kappa$ and width $W:=n l \sin (\lambda-\kappa)$ centred at the origin, as depicted in Figure 5 - that is, each point $(x, y) \in B^{ \pm}$ satisfies $\pm(x \sin \kappa+y \cos \kappa) \geq W / 2$ and $\pm(x \sin \kappa-y \cos \kappa) \geq W / 2$ (where \pm should be read consistently as + and -$)$.

Then

$$
\int_{\alpha=0}^{2 \pi}\left|\left(B^{-} \cup B^{+}\right)(\alpha)\right| \mathrm{d} \alpha \leq 8 n l-\frac{2 W^{2}}{D} .
$$

Note that $8 n l=4\left|B^{-} \cup B^{+}\right|$, so (8) is of the form (4) in Lemma 4 The proof of Lemma 7 requires a more involved argument, which will be given in Section 6 . Before that, we prove Theorems 2 and 3 using Lemmas 6 and 7

\section{Proof of Theorem 2}

We prove Theorem 2 using Lemmas 5,6 and 7 . The proof roughly goes as follows. Consider a barrier whose length is very close to 2 .

1. There cannot be too much of the barrier far outside $\square$, because that would be too wasteful by Lemma 6 .

2. This implies that there must be a significant part of the barrier near each vertex of $\square$, because this is the only place to put barrier segments that block those lines intersecting $\square$ only near this vertex.

3. Among the parts of the barrier that lie near the four vertices, there are parts that face each other and thus lead to waste by Lemma 7.

Proof of Theorem 2. Let $\square$ be the unit square, which we assume to be closed, axis-aligned, and centred at the origin. Let $B$ be its barrier. By Lemma 4 , we may assume that $B$ consists of line segments. Let $\square$ be the octagon (Figure 6 ) obtained by attaching to each edge of $\square$ an isosceles triangle of height $\frac{29}{590}$ (and thus whose identical angles are $\left.\arctan \frac{29}{295}\right)$. Let $B_{\text {out }}=B \backslash 0$. 


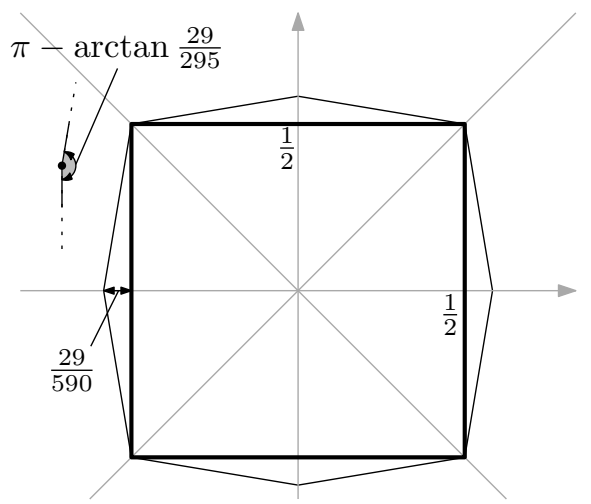

Fig. 6. Viewed from any point outside the octagon $\square$, the square $\square$ lies inside an angle that is smaller than $\pi$ by the constant $\arctan \frac{29}{295}$.

If $\left|B_{\text {out }}\right|>\frac{1}{60}$, there is a subset $B_{\text {out }}^{\prime} \subseteq B_{\text {out }}$ of length $\left|B_{\text {out }}^{\prime}\right|=\frac{1}{60}$ which is a disjoint union of finitely many line segments $b$, each lying entirely in one of the eight regions delimited by the two axes and the two bisectors of the axes. Observe that, viewed from each point on $b$, the square $\square$ lies entirely in an angle measuring $\pi-\arctan \frac{29}{295}$ (Figure 6). This allows us to apply Lemma 6 and obtain

$$
\int_{\alpha=0}^{2 \pi}|b(\alpha) \cap \square(\alpha)| \mathrm{d} \alpha \leq 4|b| \cos \left(\frac{1}{2} \arctan \frac{29}{295}\right)<4|b|-0.0048|b| .
$$

Summing up for all $b$ (and using the triangle inequality), we have

$$
\int_{\alpha=0}^{2 \pi}\left|B_{\text {out }}^{\prime}(\alpha) \cap \square(\alpha)\right| \mathrm{d} \alpha<4\left|B_{\text {out }}^{\prime}\right|-0.0048\left|B_{\text {out }}^{\prime}\right|=4\left|B_{\text {out }}^{\prime}\right|-0.00008,
$$

which yields $|B| \geq 2.00002$ by Lemma 5 . From now on, we can and will assume that $\left|B_{\text {out }}\right| \leq \frac{1}{60}$.

The intersection of $B$ and the strip $I_{0}:=\left\{(x, y) \in \mathbb{R}^{2}: \frac{7}{8} \leq x+y \leq\right.$ 1) has length at least $\sqrt{2} / 16$, because $B\left(\frac{\pi}{4}\right) \supseteq \square\left(\frac{\pi}{4}\right)=[-\sqrt{2} / 2, \sqrt{2} / 2] \supseteq$ $\left[\frac{7}{8} \sqrt{2} / 2, \sqrt{2} / 2\right]$. Let $R_{0}:=I_{0} \cap \bigcirc$ (Figure 7 ) and $B_{0}:=B \cap R_{0}$. Then we have

$$
\left|B_{0}\right|=\left|\left(B \cap I_{0}\right) \backslash B_{\text {out }}\right| \geq\left|B \cap I_{0}\right|-\left|B_{\text {out }}\right| \geq \frac{\sqrt{2}}{16}-\frac{1}{60}>0.07172=: 2 \eta .
$$

Likewise, let $R_{1}, R_{2}, R_{3}$ be the upper left, lower left, and lower right corners of $\bigcirc$, respectively. Each of the intersections $B_{1}, B_{2}, B_{3}$ of $B$ with these regions has length $>2 \eta$. Observe that $R_{0}\left(\frac{\pi}{2}-0.1813\right)$ lies above $R_{1}\left(\frac{\pi}{2}-0.1813\right)$, with a gap of size

$$
\frac{7}{8} \sin 0.1813-\left(\frac{1}{8}+2 \cdot \frac{29}{2128}\right) \cos 0.1813>0.008
$$

and $R_{0}\left(\frac{3 \pi}{4}-0.1813\right)$ lies above $R_{2}\left(\frac{3 \pi}{4}-0.1813\right)$, with an even bigger gap. 


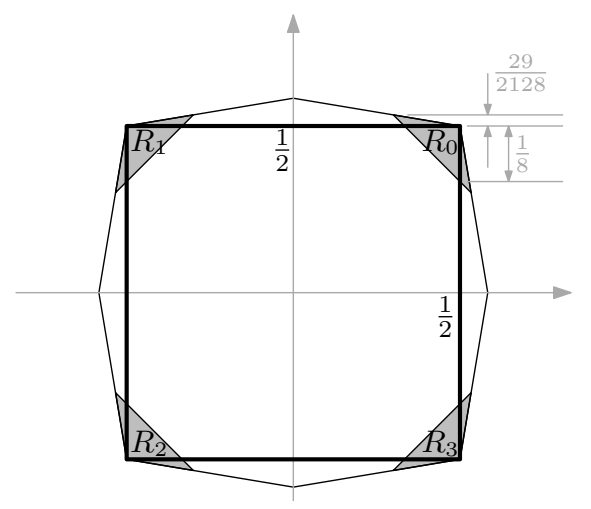

Fig. 7. The regions $R_{0}, R_{1}, R_{2}, R_{3}$.

For each $i$, we divide $B_{i}$ into three parts $B_{i, i+1}, B_{i, i+2}, B_{i, i+3}$ (the subscripts are modulo 4 ), consisting respectively of segments whose angles are in $\left[\frac{\pi}{2} i-\frac{\pi}{4}\right.$, $\left.\frac{\pi}{2} i+\frac{\pi}{8}\right),\left[\frac{\pi}{2} i+\frac{\pi}{8}, \frac{\pi}{2} i+\frac{3 \pi}{8}\right)$ and $\left[\frac{\pi}{2} i+\frac{3 \pi}{8}, \frac{\pi}{2} i+\frac{3 \pi}{4}\right)$. Thus, $B_{i, j}$ consists of segments in $B_{i}$ that "roughly point towards $R_{j}$." Since $\left|B_{i}\right|>2 \eta$, we have $\left|B_{i} \backslash B_{i, j}\right|>\eta$ for at least two of the three $j$ for each $i$, and thus, for at least eight of the twelve pairs $(i, j)$. Hence, there is $(i, j)$ such that $\left|B_{i} \backslash B_{i, j}\right|>\eta$ and $\left|B_{j} \backslash B_{j, i}\right|>\eta$.

Let $B^{-} \subseteq B_{i} \backslash B_{i, j}$ and $B^{+} \subseteq B_{j} \backslash B_{j, i}$ be finite unions of line segments of the same length such that $\left|B^{-}\right|=\left|B^{+}\right|=\eta$. Apply Lemma 7 to these $B^{-}$ and $B^{+}$, rotated and translated appropriately, and the constants $\kappa=0.1813$, $\lambda=\frac{\pi}{8}, D=\sqrt{2}$. Note that the last assumption of Lemma 7 is satisfied because $W:=\eta \sin (\lambda-\kappa)=0.03586 \sin \left(\frac{\pi}{8}-0.1813\right)=0.007524 \ldots<0.008$. This gives

$$
\int_{\alpha=0}^{2 \pi}\left|\left(B^{-} \cup B^{+}\right)(\alpha)\right| \mathrm{d} \alpha \leq 8 \eta-\frac{2 W^{2}}{D}<8 \eta-0.00008,
$$

whence $|B| \geq 2.00002$ by Lemma 5

\section{Proof of Theorem 3}

Theorem 3 is proved by modifying the proof of Theorem 2 (Section 4 ) as follows. Let $x_{i}$ be distinct points $(i=1,2,3,4)$ on the boundary of $U$ at which $U$ is strictly convex, i.e., there is a line that intersects $U$ only at $x_{i}$; let $\alpha_{i}$ be the angle of this line. Note that such four points exist unless $U$ is a triangle. Let $R_{i}$ be a sufficiently small closed neighbourhood of $x_{i}$, so that no three of $R_{1}, R_{2}$, $R_{3}, R_{4}$ are stabbed by a line.

Instead of the octagon $\square$, we consider the set $S_{\delta} \supseteq U$ of points such that a random line through this point avoids $U$ with probability less than a positive constant $\delta$ (Figure 8). By applying Lemma 6 in the same way (with some routine compactness argument), we know that $B_{\text {out }}:=B \backslash S_{\delta}$ must be small (under the 


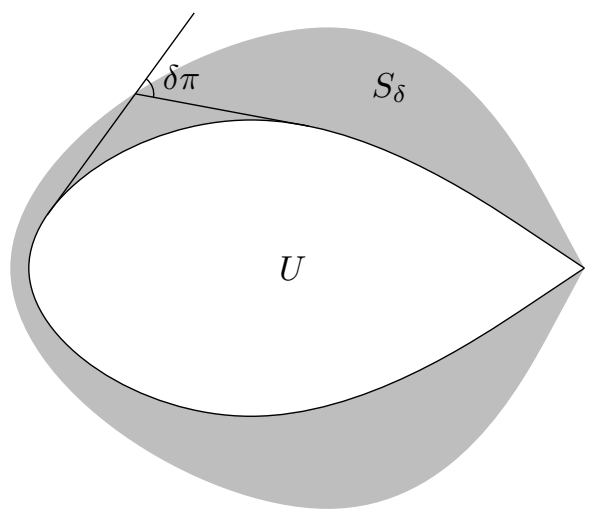

Fig. 8. $S_{\delta}$ is the set of points from which $U$ looks big. Putting too much of the barrier outside $S_{\delta}$ is wasteful.

assumption of $|B| \leq p+\varepsilon$, for an appropriately small $\varepsilon$ ). By taking $\delta$ sufficiently small, $S_{\delta}$ comes so close to $U$ that the following happens for each $i=1,2,3,4$ : there is a neighbourhood $N \subseteq U$ of $x_{i}$ in $U$ such that every angle- $\alpha_{i}$ line that intersects $N$ intersects $S_{\delta}$ only in $R_{i}$. This guarantees that the part $B_{i}:=B \cap R_{i}$ of the barrier must have length at least some positive constant (just to block those angle- $\alpha_{i}$ lines that hit $N$ ). This allows us to define $B_{i, j}$ in the way similar to Theorem 2 and apply Lemma 7 with appropriate $\kappa, \lambda, D$.

This proves Theorem 3. To see that the constant $\varepsilon$ in the statement must depend on $U$, just consider arbitrarily thin rectangles.

\section{Proof of Lemma 7}

It remains to prove Lemma 7. Let us first interpret what it roughly claims. By symmetry, we can halve the interval $[0,2 \pi]$ and replace $(8)$ by

$$
4 n l-\int_{\alpha=0}^{\pi}\left|\left(B^{-} \cup B^{+}\right)(\alpha)\right| \mathrm{d} \alpha \geq \frac{W^{2}}{D} .
$$

Let $\mathcal{B}^{-}$and $\mathcal{B}^{+}$be the sets of line segments of length $l$ comprising $B^{-}$and $B^{+}$, respectively. For each $b \in \mathcal{B}^{-} \cup \mathcal{B}^{+}$, consider the region

$$
R_{b}:=\{(\alpha, v) \in[0, \pi] \times \mathbb{R}: v \in b(\alpha)\},
$$

whose area is $2 l$. Note that the first term $4 n l$ of $(14)$ is the sum of this area for all $b \in \mathcal{B}^{-} \cup \mathcal{B}^{+}$, whereas the second term is the area of the union. Thus, (14) says that the area of the overlap (considering multiplicity) is at least $W^{2} / D$. Since this term $W^{2} / D$ is proportional to $n^{2}$, which is the number of pairs $\left(b, b^{\prime}\right) \in \mathcal{B}^{-} \times \mathcal{B}^{+}$, we should lower-bound (by a constant determined by $\lambda, \kappa, D$ ) the area of the 


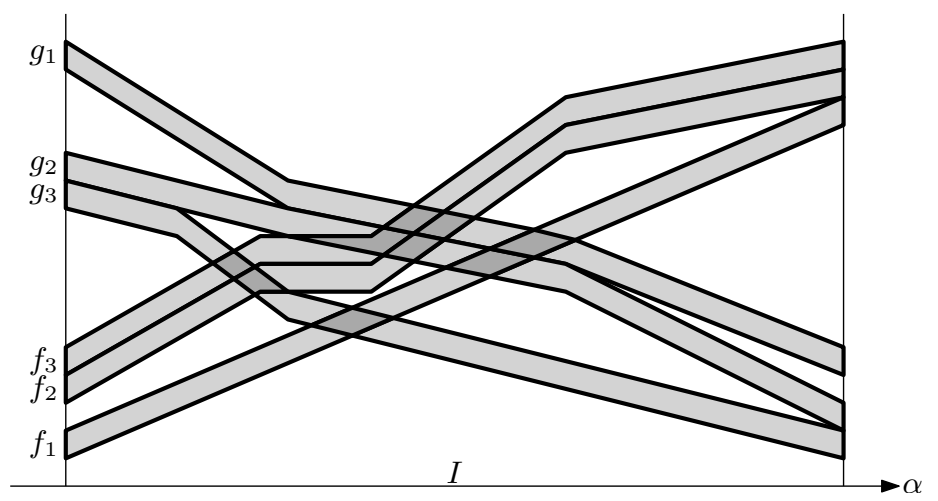

Fig. 9. Lemma 8 is easy if $\left\{f_{1}, \ldots, f_{n}\right\}$ and $\left\{g_{1}, \ldots, g_{n}\right\}$ are simple. In this case, we only have overlaps of the form $R_{f_{i}} \cap R_{g_{j}}$ (nine dark regions in the figure), and we can underestimate their areas separately. We reduce the general case to this easy case.

overlap $R_{b} \cap R_{b^{\prime}}$ per such pair $\left(b, b^{\prime}\right)$. This is relatively easy if the overlaps $R_{b} \cap R_{b^{\prime}}$ are all disjoint (using the fact that $R_{b}$ and $R_{b^{\prime}}$ must cross roughly in the middle because of the configuration in Figure 5), but it can get tricky otherwise.

To analyze such a situation, we start with the following lemma, which makes a similar estimate on the size of potentially complicated overlaps, but of simpler objects, namely bands with constant width.

Lemma 8. Let $I \subseteq \mathbb{R}$ be an interval and let $W, D \geq 0$. Let $\mathcal{U}$ be the set of functions $f$ which take each $\alpha \in I$ to an interval $f(\alpha)=[f(\alpha), \bar{f}(\alpha)]$ of length $W / n$ and are $\frac{1}{2} D$-Lipschitz, that is, $\left|f\left(\alpha_{0}\right)-f\left(\alpha_{1}\right)\right| \leq \frac{1}{2} D \cdot\left|\alpha_{0}-\alpha_{1}\right|$ for each $\alpha_{0}, \alpha_{1} \in I$. Suppose that $2 n$ functions $\bar{f}_{1}, \ldots, \bar{f}_{n}, g_{1}, \ldots, g_{n} \in \mathcal{U}$ satisfy

$$
\underline{g_{j}}(\min I)-\overline{f_{i}}(\min I) \geq W, \quad \underline{f_{i}}(\max I)-\overline{g_{j}}(\max I) \geq W
$$

for each $i, j$ (i.e., the functions $f_{i}$ start far below $g_{j}$ and end up far above). Then

$$
\left|R_{f_{1}} \cup \cdots \cup R_{f_{n}} \cup R_{g_{1}} \cup \cdots \cup R_{g_{n}}\right| \leq 2 W|I|-\frac{W^{2}}{D},
$$

where $R_{f}:=\{(\alpha, v) \in I \times \mathbb{R}: v \in f(\alpha)\}$ denotes the graph of $f \in \mathcal{U}$.

Proof. Since $\left|R_{f}\right|=|I| \cdot W / n$ for each $f \in \mathcal{U}$, the bound (17) says that the loss of area caused by overlaps is at least $W^{2} / D$.

We say that $\left\{f_{1}, \ldots, f_{n}\right\} \subseteq \mathcal{U}$ is simple if $R_{f_{i}} \cap R_{f_{j}}=\emptyset$ for all distinct $i, j$. If both $\left\{f_{1}, \ldots, f_{n}\right\}$ and $\left\{g_{1}, \ldots, g_{n}\right\}$ are simple (Figure 9), we easily get (17), because in this case, the $n^{2}$ overlaps $R_{f_{i}} \cap R_{g_{j}}$ are all disjoint, and each of them has area $\geq(W / n)^{2} / D$. In fact, instead of $(16)$, it would have sufficed to assume

$$
\underline{g_{j}}(\min I) \geq \overline{f_{i}}(\min I), \quad \underline{f_{i}}(\max I) \geq \overline{g_{j}}(\max I) .
$$


We will reduce the general case to this easy special case. That is, starting from $\left\{f_{1}, \ldots, f_{n}\right\}$ and $\left\{g_{1}, \ldots, g_{n}\right\}$ that satisfy (16), we define simple $\left\{f_{1}^{\prime}, \ldots, f_{n}^{\prime}\right\}$ and $\left\{g_{1}^{\prime}, \ldots, g_{n}^{\prime}\right\}$ satisfying (18), such that $R_{f_{1}^{\prime}} \cup \cdots \cup R_{f_{n}^{\prime}} \supseteq R_{f_{1}} \cup \cdots \cup R_{f_{n}}$ and $R_{g^{\prime}} \cup \cdots \cup R_{g_{n}^{\prime}} \supseteq R_{g_{1}} \cup \cdots \cup R_{g_{n}}$ (note that by these containments, the bound (17) for the $f_{i}^{\prime}$ and the $g_{j}^{\prime}$ implies (17) for the $f_{i}$ and the $g_{j}$ ).

We describe how we modify $\left\{f_{1}, \ldots, f_{n}\right\}$ to obtain $\left\{f_{1}^{\prime}, \ldots, f_{n}^{\prime}\right\}$ (modification on $\left\{g_{1}, \ldots, g_{n}\right\}$ is done similarly and independently). First, we make sure that the functions $f_{i}$ never switch relative positions, by exchanging the roles of two intervals at every time $\alpha$ at which one overtakes another. This way, we ensure that at each time $\alpha$, the intervals $f_{1}(\alpha), \ldots, f_{n}(\alpha)$ are in ascending order.

These intervals may still overlap one another. So we push them upwards one by one as necessary to avoid previous intervals. Specifically, define $f_{1}^{\prime}$ by $f_{1}^{\prime}(\alpha)=f_{1}(\alpha)$, and then $f_{i}^{\prime}$ for $i=2,3, \ldots$ by $f_{i}^{\prime}(\alpha)=\max \left\{\underline{f_{i}}(\alpha)\right.$, max $\left.\overline{f_{i-1}^{\prime}}(\alpha)\right\}$.

The functions remain $\frac{1}{2} D$-Lipschitz, since the resulting $f_{i}^{\prime}$ at each time $\alpha$ moves at the same speed as one of the original $f_{j}$. The condition 18 is also satisfied, since initially we had (16) and then we moved each $f_{i}^{\prime}$ upwards by at most $W$.

Proof of Lemma 7, Let $\mathcal{B}^{-}, \mathcal{B}^{+}$and $R_{b}$ be as at the beginning of Section 6 . As explained there, our goal it to prove (14), which says that the area of the overlap between $R_{b}$ for $b \in \mathcal{B}^{-} \cup \mathcal{B}^{+}$is at least $W^{2} / D$. We claim that this is true even if we replace each $R_{b}$ by its subset $\tilde{R}_{b}$ defined below.

Let $I:=\left[\frac{\pi}{2}-\kappa, \frac{\pi}{2}+\kappa\right]$. Note that, because of the configuration of segments (Figure 5), we have $|b(\alpha)| \geq l \sin (\lambda-\kappa)=W / n$ for each $\alpha \in I$ and $b \in \mathcal{B}^{-} \cup \mathcal{B}^{+}$. We define a subset of $R_{b}$ (see (15) by restricting $\alpha$ to $I$ and replacing the interval $b(\alpha)$ by its subinterval $\tilde{b}(\alpha):=[\min (b(\alpha)), \min (b(\alpha))+W / n]$ :

$$
\tilde{R}_{b}:=\{(\alpha, v) \in I \times \mathbb{R}: v \in \tilde{b}(\alpha)\} .
$$

Our claim was that the total area of pairwise overlaps between $\tilde{R}_{b}$ for $b \in \mathcal{B}^{-} \cup \mathcal{B}^{+}$ is at least $W^{2} / D$. But this is Lemma 8 applied to $\left\{f_{1}, \ldots, f_{n}\right\}=\left\{\tilde{b}: b \in \mathcal{B}^{-}\right\}$, $\left\{g_{1}, \ldots, g_{n}\right\}=\left\{\tilde{b}: b \in \mathcal{B}^{+}\right\}$.

\section{Half-line barriers}

Let us finally propose an analogous question, obtained by replacing lines by halflines in the definition of barriers: a set $B \subseteq \mathbb{R}^{2}$ is a half-line barrier of $U \subseteq \mathbb{R}^{2}$ if all half-lines intersecting $U$ intersect $B$. This intuitively means "hiding the object $U$ from outside," which we find perhaps as natural, if not more, than the notion of opaque sets. Similarly to Lemma 1, we have

Lemma 9. $|B| \geq p$ for any rectifiable half-line barrier $B$ of a convex set $U \subseteq \mathbb{R}^{2}$ with perimeter $p$.

Thus, unlike for line barriers, the question is completely answered when $U$ is connected: the shortest half-line barrier is the boundary of the convex hull. 


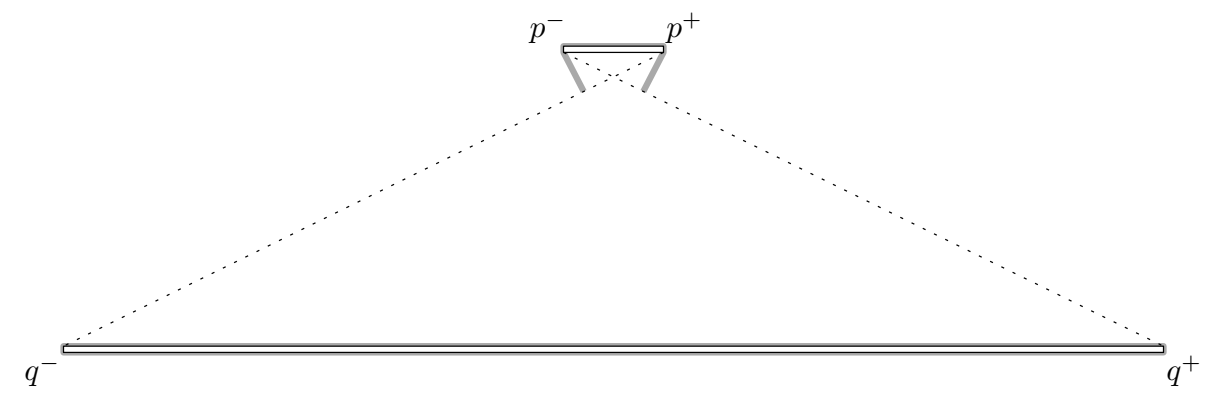

Fig. 10. Consider the line segments $p^{-} p^{+}$and $q^{-} q^{+}$, where $p^{ \pm}=( \pm 1,8)$ and $q^{ \pm}=$ $( \pm 15,0)$, and let $U$ be the union of these segments with small "thickness": $U$ consists of a rectangle with vertices $( \pm 1,8 \pm \varepsilon)$ and another with vertices $( \pm 15, \pm \varepsilon)$, for a small $\varepsilon>0$. The boundaries of these thick line segments have total length 64 (plus a small amount due to the thickness). The boundary of the convex hull of all of $U$ has length $2+30+2 \sqrt{260}>64.24$ (plus thickness). But we have another half-line barrier depicted above in gray, whose total length is $2+60+2 / \sqrt{5}+2 / \sqrt{5}<63.79$ (plus thickness, which can be made arbitrarily small).

If $U$ is disconnected, there can be shorter half-line barriers. For example, if $U$ consists of two connected components that are enough far apart from each other, it is more efficient to cover them separately than together. One might hope that an optimal half-line barrier is always obtained by grouping the connected components of $U$ in some way and taking convex hulls of each. This is not true, as the example in Figure 10 shows. We have not been able to find an algorithm that achieves a nontrivial approximation ratio for this problem.

Acknowledgements. We are grateful to Gábor Tardos for many interesting discussions on the subject. In particular, the present proof of Lemma 4 is based on his idea.

\section{References}

[1] H. T. Croft. Curves intersecting certain sets of great-circles on the sphere. Journal of the London Mathematical Society (2), 1, 461-469, 1969.

[2] E. Demaine and J. O'Rourke. Open problems from CCCG 2007. In Proc. 20th Canadian Conference on Computational Geometry (CCCG 2008), 183-186, 2008.

[3] A. Dumitrescu and M. Jiang. The opaque square. Preprint: arXiv:1311.3323v1. Accepted for presentation at the 30th Annual Symposium on Computational Geometry (SoCG 2014).

[4] A. Dumitrescu and M. Jiang. Computational Geometry Column 58. SIGACT News, 44(4), 73-78, 2013.

[5] A. Dumitrescu, M. Jiang, and J. Pach. Opaque sets. Algorithmica, in press. Preliminary version in the Proc. 14th International Workshop on Approximation Algorithms for Combinatorial Optimization Problems (APPROX 2011), LNCS 6845, 194-205, 2011. Preprint: arXiv:1005.2218v5. 
[6] V. Faber and J. Mycielski. The shortest curve that meets all the lines that meet a convex body. American Mathematical Monthly, 93, 796-801, 1986.

[7] V. Faber, J. Mycielski, and P. Pedersen. On the shortest curve which meets all the lines which meet a circle. Annales Polonici Mathematici, 44, 249-266, 1984.

[8] R. E. D. Jones. Opaque sets of degree $\alpha$. American Mathematical Monthly, 71, $535-537,1964$

[9] B. Kawohl. The opaque square and the opaque circle. General Inequalities 7, ISNM International Series of Numerical Mathematics Volume 123, 339-346, 1997.

[10] B. Kawohl. Some nonconvex shape optimization problems. In Optimal Shape Design, 7-46, Springer, 2000.

[11] S. Mazurkiewicz. Przykład zbioru domkniętego, punktokształtnego, mającego punkty wspólne z każdą prostą, przecinającą pewien obszar domknięty. (Sur un ensemble fermé, punctiforme, qui rencontre toute droite passant par un certain domaine.) Prace Matematyczno-Fizyczne, 27, 11-16, 1916. In Polish (French summary).

[12] J.S. Provan, M. Brazil, D. Thomas, J.F. Weng. Minimum opaque covers for polygonal regions. Preprint: $\operatorname{arXiv:1210.8139} 1$. 